\title{
Arithmetic Properties of a Restricted Bipartition Function
}

\author{
Jian Liu \\ School of Insurance \\ Central University of Finance and Economics \\ Beijing 102206, P.R. China \\ liujian8210@gmail.com \\ Andrew Y.Z. Wang* \\ School of Mathematical Sciences \\ University of Electronic Science and Technology of China \\ Chengdu 611731, P.R. China \\ yzwang@uestc.edu.cn
}

Submitted: Feb 12, 2015; Accepted: Jun 19, 2015; Published: Jul 1, 2015

Mathematics Subject Classifications: 05A17, 11P83

\begin{abstract}
A bipartition of $n$ is an ordered pair of partitions $(\lambda, \mu)$ such that the sum of all of the parts equals $n$. In this article, we concentrate on the function $c_{5}(n)$, which counts the number of bipartitions $(\lambda, \mu)$ of $n$ subject to the restriction that each part of $\mu$ is divisible by 5 . We explicitly establish four Ramanujan type congruences and several infinite families of congruences for $c_{5}(n)$ modulo 3 .
\end{abstract}

Keywords: bipartition, congruence

\section{Introduction}

In a series of papers $[4,5,6]$, Chan studied the arithmetic properties of the cubic partition function $a(n)$, which is defined by

$$
\sum_{n=0}^{\infty} a(n) q^{n}=\frac{1}{(q ; q)_{\infty}\left(q^{2} ; q^{2}\right)_{\infty}}
$$

\footnotetext{
*Corresponding author
} 
Throughout the paper, we adopt the following standard $q$-series notation

$$
(a ; q)_{\infty}=\prod_{n=1}^{\infty}\left(1-a q^{n-1}\right) .
$$

In [4], Chan proved that

Theorem 1. For $n \geqslant 0$,

$$
a(3 n+2) \equiv 0 \quad(\bmod 3) .
$$

Later, Kim [13] gave a combinatorial interpretation of the congruence (1). Furthermore, Chan [5] showed that

Theorem 2. For $k \geqslant 1$ and $n \geqslant 0$,

$$
a\left(3^{k} n+s_{k}\right) \equiv 0 \quad\left(\bmod 3^{k+\delta(k)}\right),
$$

where $s_{k}$ is the reciprocal modulo $3^{k}$ of 8 and $\delta(k)=1$ if $k$ is even, and 0 otherwise.

Chan and Toh [7] also established the following nice congruence, which was also discovered by Xiong [20] independently.

Theorem 3. If $k \geqslant 1$ and $n \geqslant 0$, then

$$
a\left(5^{k} n+t_{k}\right) \equiv 0 \quad\left(\bmod 5^{\lfloor k / 2\rfloor}\right),
$$

where $t_{k}$ is the reciprocal modulo $5^{k}$ of 8 .

Inspired by the work of Ramanujan on the standard partition function $p(n)$, Chan [5] asked whether there are any other congruences of the following form

$$
a(\ell n+k) \equiv 0 \quad(\bmod \ell),
$$

where $\ell$ is prime and $0 \leqslant k<\ell$. Later, Sinick [18] answered Chan's question in the negative by considering the following restricted bipartition function:

$$
\sum_{n=0}^{\infty} c_{N}(n) q^{n}=\frac{1}{(q ; q)_{\infty}\left(q^{N} ; q^{N}\right)_{\infty}} .
$$

A bipartition of $n$ is an ordered pair of partitions $(\lambda, \mu)$ such that the sum of all of the parts equals $n$. Then we know that $c_{N}(n)$ counts the number of bipartitions $(\lambda, \mu)$ of $n$ subject to the restriction that each part of $\mu$ is divisible by $N$. Recently, bipartitions with certain restrictions on each partition have been investigated by many authors, see $[3,8,9,10,11,12,14,15,16,17,19]$ for instance.

In this paper, we investigate the bipartition function $c_{5}(n)$ from an arithmetic point of view in the spirit of Ramanujan's congruences for the standard partition function $p(n)$. 


\section{Ramanujan type congruences for $c_{5}(n)$}

We first introduce a useful lemma which will be used later.

Lemma 4. We have

$$
(q ; q)_{\infty}^{2}\left(q^{5} ; q^{5}\right)_{\infty}^{2} \equiv\left(q^{3} ; q^{3}\right)_{\infty}^{4}+q\left(q^{3} ; q^{3}\right)_{\infty}^{2}\left(q^{15} ; q^{15}\right)_{\infty}^{2}-q^{2}\left(q^{15} ; q^{15}\right)_{\infty}^{4} \quad(\bmod 3) .
$$

Proof. From [1, p.28, Entry 1.6.2], we see that

$$
\begin{aligned}
(q ; q)_{\infty}^{2}\left(q^{5} ; q^{5}\right)_{\infty}^{2} & =\left(\psi^{2}(q)-q \psi^{2}\left(q^{5}\right)\right)\left(\psi^{2}(q)-5 q \psi^{2}\left(q^{5}\right)\right) \\
& \equiv \psi(q) \psi\left(q^{3}\right)-q^{2} \psi\left(q^{5}\right) \psi\left(q^{15}\right)(\bmod 3)
\end{aligned}
$$

where

$$
\psi(q)=\sum_{n=0}^{\infty} q^{n(n+1) / 2}=\frac{\left(q^{2} ; q^{2}\right)_{\infty}^{2}}{(q ; q)_{\infty}} .
$$

Invoking [2, p.49, Corollary (ii)], we have

$$
\psi(q)=A\left(q^{3}\right)+q \psi\left(q^{9}\right)
$$

where

$$
A(q)=\left(-q ; q^{3}\right)_{\infty}\left(-q^{2} ; q^{3}\right)_{\infty}\left(q^{3} ; q^{3}\right)_{\infty} .
$$

Substituting (5) into (4), we find that

$$
\begin{aligned}
(q ; q)_{\infty}^{2}\left(q^{5} ; q^{5}\right)_{\infty}^{2} \equiv & \psi\left(q^{3}\right)\left(A\left(q^{3}\right)+q \psi\left(q^{9}\right)\right) \\
& -q^{2} \psi\left(q^{15}\right)\left(A\left(q^{15}\right)+q^{5} \psi\left(q^{45}\right)\right) \quad(\bmod 3) \\
= & \psi\left(q^{3}\right) A\left(q^{3}\right)-q^{2} \psi\left(q^{15}\right) A\left(q^{15}\right) \\
& +q\left(\psi\left(q^{3}\right) \psi\left(q^{9}\right)-q^{6} \psi\left(q^{15}\right) \psi\left(q^{45}\right)\right) .
\end{aligned}
$$

On the other hand, applying (4) with $q$ replaced by $q^{3}$ yields that

$$
\left(q^{3} ; q^{3}\right)_{\infty}^{2}\left(q^{15} ; q^{15}\right)_{\infty}^{2} \equiv \psi\left(q^{3}\right) \psi\left(q^{9}\right)-q^{6} \psi\left(q^{15}\right) \psi\left(q^{45}\right) \quad(\bmod 3)
$$

Therefore, we arrive at

$$
(q ; q)_{\infty}^{2}\left(q^{5} ; q^{5}\right)_{\infty}^{2} \equiv \psi\left(q^{3}\right) A\left(q^{3}\right)-q^{2} \psi\left(q^{15}\right) A\left(q^{15}\right)+q\left(q^{3} ; q^{3}\right)_{\infty}^{2}\left(q^{15} ; q^{15}\right)_{\infty}^{2} \quad(\bmod 3)
$$

In addition, it is easy to see that

$$
\psi(q) A(q) \equiv(q ; q)_{\infty}^{4} \quad(\bmod 3)
$$

Utilizing the above congruence in (6), we complete the proof of (3).

With Lemma 4 in hand, we now move to the dissections of the generating function for $c_{5}(n)$ modulo 3. 
Theorem 5. We have

$$
\begin{aligned}
\sum_{n=0}^{\infty} c_{5}(3 n) q^{n} & \equiv \frac{\left(q^{3} ; q^{3}\right)_{\infty}}{\left(q^{5} ; q^{5}\right)_{\infty}} \quad(\bmod 3) \\
\sum_{n=0}^{\infty} c_{5}(3 n+1) q^{n} & \equiv(q ; q)_{\infty}\left(q^{5} ; q^{5}\right)_{\infty} \quad(\bmod 3) \\
\sum_{n=0}^{\infty} c_{5}(3 n+2) q^{n} & \equiv-\frac{\left(q^{15} ; q^{15}\right)_{\infty}}{(q ; q)_{\infty}}(\bmod 3)
\end{aligned}
$$

Proof. From (2), we can easily deduce that

$$
\sum_{n=0}^{\infty} c_{5}(n) q^{n} \equiv \frac{(q ; q)_{\infty}^{2}\left(q^{5} ; q^{5}\right)_{\infty}^{2}}{\left(q^{3} ; q^{3}\right)_{\infty}\left(q^{15} ; q^{15}\right)_{\infty}} \quad(\bmod 3) .
$$

Applying Lemma 4, we obtain

$$
\begin{aligned}
\sum_{n=0}^{\infty} c_{5}(n) q^{n} & \equiv \frac{\left(q^{3} ; q^{3}\right)_{\infty}^{3}}{\left(q^{15} ; q^{15}\right)_{\infty}}+q\left(q^{3} ; q^{3}\right)_{\infty}\left(q^{15} ; q^{15}\right)_{\infty}-q^{2} \frac{\left(q^{15} ; q^{15}\right)_{\infty}^{3}}{\left(q^{3} ; q^{3}\right)_{\infty}} \\
& \equiv \frac{\left(q^{9} ; q^{9}\right)_{\infty}}{\left(q^{15} ; q^{15}\right)_{\infty}}+q\left(q^{3} ; q^{3}\right)_{\infty}\left(q^{15} ; q^{15}\right)_{\infty}-q^{2} \frac{\left(q^{45} ; q^{45}\right)_{\infty}}{\left(q^{3} ; q^{3}\right)_{\infty}} \quad(\bmod 3)
\end{aligned}
$$

frow which we get

$$
\begin{aligned}
\sum_{n=0}^{\infty} c_{5}(3 n) q^{3 n} & \equiv \frac{\left(q^{9} ; q^{9}\right)_{\infty}}{\left(q^{15} ; q^{15}\right)_{\infty}}(\bmod 3), \\
\sum_{n=0}^{\infty} c_{5}(3 n+1) q^{3 n+1} & \equiv q\left(q^{3} ; q^{3}\right)_{\infty}\left(q^{15} ; q^{15}\right)_{\infty} \quad(\bmod 3), \\
\sum_{n=0}^{\infty} c_{5}(3 n+2) q^{3 n+2} & \equiv-q^{2} \frac{\left(q^{45} ; q^{45}\right)_{\infty}}{\left(q^{3} ; q^{3}\right)_{\infty}}(\bmod 3),
\end{aligned}
$$

simplification upon which yields the desired results.

The following is a consequence of Theorem 5 .

Corollary 6. We have

$$
\sum_{n=0}^{\infty} c_{5}(9 n+7) q^{n} \equiv-\left(q^{3} ; q^{3}\right)_{\infty}\left(q^{15} ; q^{15}\right)_{\infty} \quad(\bmod 3) .
$$


Proof. By (8), we find that

$$
\begin{aligned}
\sum_{n=0}^{\infty} c_{5}(3 n+1) q^{n} \equiv & \left(q^{3} ; q^{3}\right)_{\infty}\left(q^{15} ; q^{15}\right)_{\infty} \times \frac{1}{(q ; q)_{\infty}^{2}\left(q^{5} ; q^{5}\right)_{\infty}^{2}} \quad(\bmod 3) \\
= & \left(q^{3} ; q^{3}\right)_{\infty}\left(q^{15} ; q^{15}\right)_{\infty} \times\left(\sum_{n=0}^{\infty} c_{5}(3 n) q^{3 n}\right. \\
& \left.+\sum_{n=0}^{\infty} c_{5}(3 n+1) q^{3 n+1}+\sum_{n=0}^{\infty} c_{5}(3 n+2) q^{3 n+2}\right)^{2}
\end{aligned}
$$

Extracting those terms on each side for which the powers of $q$ are of the form $3 n+2$, dividing by $q^{2}$, and replacing $q^{3}$ by $q$, we obtain

$$
\begin{aligned}
\sum_{n=0}^{\infty} c_{5}(9 n+7) q^{n} \equiv & (q ; q)_{\infty}\left(q^{5} ; q^{5}\right)_{\infty} \times\left(\left(\sum_{n=0}^{\infty} c_{5}(3 n+1) q^{n}\right)^{2}\right. \\
& \left.+2 \sum_{n=0}^{\infty} c_{5}(3 n) q^{n} \sum_{n=0}^{\infty} c_{5}(3 n+2) q^{n}\right) \quad(\bmod 3)
\end{aligned}
$$

It follows from Theorem 5 that

$$
\begin{aligned}
\sum_{n=0}^{\infty} c_{5}(9 n+7) q^{n} & \equiv(q ; q)_{\infty}\left(q^{5} ; q^{5}\right)_{\infty} \times\left((q ; q)_{\infty}^{2}\left(q^{5} ; q^{5}\right)_{\infty}^{2}-2 \frac{\left(q^{3} ; q^{3}\right)_{\infty}\left(q^{15} ; q^{15}\right)_{\infty}}{\left(q^{5} ; q^{5}\right)_{\infty}(q ; q)_{\infty}}\right) \\
& \equiv-\left(q^{3} ; q^{3}\right)_{\infty}\left(q^{15} ; q^{15}\right)_{\infty} \quad(\bmod 3) .
\end{aligned}
$$

This completes the proof.

We now establish four Ramanujan type congruences for $c_{5}(n)$.

Theorem 7. For all $n \geqslant 0$,

$$
\begin{aligned}
c_{5}(15 n+6) & \equiv 0 \quad(\bmod 3), \\
c_{5}(15 n+10) & \equiv 0 \quad(\bmod 3), \\
c_{5}(15 n+12) & \equiv 0 \quad(\bmod 3), \\
c_{5}(15 n+13) & \equiv 0 \quad(\bmod 3) .
\end{aligned}
$$

Proof. Recall that Euler's pentagonal number theorem [2, p.36, Entry 22]

$$
(q ; q)_{\infty}=\sum_{n=-\infty}^{\infty}(-1)^{n} q^{n(3 n+1) / 2} .
$$


Substituting (15) into (7), we have

$$
\sum_{n=0}^{\infty} c_{5}(3 n) q^{n} \equiv \frac{1}{\left(q^{5} ; q^{5}\right)_{\infty}} \sum_{n=-\infty}^{\infty}(-1)^{n} q^{3 n(3 n+1) / 2} \quad(\bmod 3) .
$$

Extracting those terms on each side whose power of $q$ is of the form $5 n+2$ or $5 n+4$, and employing the fact that there exist no integers $n$ such that $3 n(3 n+1) / 2$ is congruent to 2 or 4 modulo 5 , we get

$$
\sum_{n=0}^{\infty} c_{5}(15 n+6) q^{5 n+2} \equiv \sum_{n=0}^{\infty} c_{5}(15 n+12) q^{5 n+4} \equiv 0 \quad(\bmod 3),
$$

which means that

$$
c_{5}(15 n+6) \equiv c_{5}(15 n+12) \equiv 0 \quad(\bmod 3) .
$$

Similarly, from (8) and the fact that there are no integers $n$ with $n(3 n+1) / 2$ being congruent to 3 or 4 modulo 5 , it is not hard to obtain

$$
c_{5}(15 n+10) \equiv c_{5}(15 n+13) \equiv 0 \quad(\bmod 3) .
$$

This concludes the proof.

\section{Two infinite families of congruences for $c_{5}(n)$}

We start with investigating a generalization of the congruences (8) and (10).

Theorem 8. For $\alpha \geqslant 1$, we have

$$
\begin{aligned}
\sum_{n=0}^{\infty} c_{5}\left(3^{2 \alpha-1} n+\frac{3^{2 \alpha-1}+1}{4}\right) q^{n} & \equiv(-1)^{\alpha+1}(q ; q)_{\infty}\left(q^{5} ; q^{5}\right)_{\infty} \quad(\bmod 3), \\
\sum_{n=0}^{\infty} c_{5}\left(3^{2 \alpha} n+\frac{3^{2 \alpha+1}+1}{4}\right) q^{n} & \equiv(-1)^{\alpha}\left(q^{3} ; q^{3}\right)_{\infty}\left(q^{15} ; q^{15}\right)_{\infty} \quad(\bmod 3) .
\end{aligned}
$$

Proof. We proceed by induction on $\alpha$. The case $\alpha=1$ corresponds to the congruences (8) and (10).

Assume that

$$
\sum_{n=0}^{\infty} c_{5}\left(3^{2 \alpha} n+\frac{3^{2 \alpha+1}+1}{4}\right) q^{n} \equiv(-1)^{\alpha}\left(q^{3} ; q^{3}\right)_{\infty}\left(q^{15} ; q^{15}\right)_{\infty} \quad(\bmod 3)
$$

is true for some fixed integer $\alpha \geqslant 1$. Since the terms appearing on the right side of the above congruence are powers of $q^{3}$, we have

$$
\sum_{n=0}^{\infty} c_{5}\left(3^{2 \alpha}(3 n)+\frac{3^{2 \alpha+1}+1}{4}\right) q^{3 n} \equiv(-1)^{\alpha}\left(q^{3} ; q^{3}\right)_{\infty}\left(q^{15} ; q^{15}\right)_{\infty} \quad(\bmod 3),
$$


which yields that

$$
\sum_{n=0}^{\infty} c_{5}\left(3^{2 \alpha+1} n+\frac{3^{2 \alpha+1}+1}{4}\right) q^{n} \equiv(-1)^{\alpha+2}(q ; q)_{\infty}\left(q^{5} ; q^{5}\right)_{\infty} \quad(\bmod 3) .
$$

Now we suppose that

$$
\sum_{n=0}^{\infty} c_{5}\left(3^{2 \alpha-1} n+\frac{3^{2 \alpha-1}+1}{4}\right) q^{n} \equiv(-1)^{\alpha+1}(q ; q)_{\infty}\left(q^{5} ; q^{5}\right)_{\infty} \quad(\bmod 3)
$$

is true for some fixed integer $\alpha \geqslant 1$, to which applying the same argument as in the proof of Corollary 6 yields that

$$
\sum_{n=0}^{\infty} c_{5}\left(3^{2 \alpha} n+\frac{3^{2 \alpha+1}+1}{4}\right) q^{n} \equiv(-1)^{\alpha+2}\left(q^{3} ; q^{3}\right)_{\infty}\left(q^{15} ; q^{15}\right)_{\infty} \quad(\bmod 3) .
$$

The proof is complete.

As a consequence of Theorem 8, we have the following result.

Corollary 9. If $\alpha \geqslant 1$ and $n \geqslant 0$,

$$
\begin{aligned}
c_{5}\left(3^{2 \alpha+1} n+\frac{7 \times 3^{2 \alpha}+1}{4}\right) & \equiv 0(\bmod 3), \\
c_{5}\left(3^{2 \alpha+1} n+\frac{11 \times 3^{2 \alpha}+1}{4}\right) & \equiv 0 \quad(\bmod 3) .
\end{aligned}
$$

Proof. Note that all the terms on the right hand side of (17) are of the form $q^{3 n}$. We can immediately obtain (18) and (19) by equating the coefficients of $q^{3 n+1}$ and $q^{3 n+2}$ on both sides of (17).

\section{More infinite families of congruences for $c_{5}(n)$}

To establish new congruences for $c_{5}(n)$, we need the following lemma.

Lemma 10. Let

$$
\sum_{n=0}^{\infty} b(n) q^{n}=(q ; q)_{\infty}\left(q^{5} ; q^{5}\right)_{\infty}
$$

Then, for a given prime $p \geqslant 5$ with $\left(\frac{-5}{p}\right)=-1$, we have

$$
\sum_{n=0}^{\infty} b\left(p n+\frac{p^{2}-1}{4}\right) q^{n}=\left(q^{p} ; q^{p}\right)_{\infty}\left(q^{5 p} ; q^{5 p}\right)_{\infty}
$$


Proof. Applying Euler's pentagonal number theorem, we have

$$
\sum_{n=0}^{\infty} b(n) q^{n}=\sum_{m, n=-\infty}^{\infty}(-1)^{m+n} q^{m(3 m+1) / 2+5 n(3 n+1) / 2} .
$$

We now consider

$$
\frac{m(3 m+1)}{2}+\frac{5 n(3 n+1)}{2} \equiv \frac{p^{2}-1}{4}(\bmod p)
$$

namely,

$$
(6 m+1)^{2}+5(6 n+1)^{2} \equiv 0 \quad(\bmod p) .
$$

Since $\left(\frac{-5}{p}\right)=-1$, we deduce that

$$
6 m+1 \equiv 6 n+1 \equiv 0 \quad(\bmod p) .
$$

If $p \equiv 1(\bmod 6)$, then $m \equiv n \equiv \frac{p-1}{6}(\bmod p)$. Let

$$
m=k p+\frac{p-1}{6} \text { and } n=l p+\frac{p-1}{6},
$$

we have

$$
m(3 m+1) / 2+5 n(3 n+1) / 2=\left(p^{2}-1\right) / 4+p^{2}\left(3 k^{2}+k\right) / 2+5 p^{2}\left(3 l^{2}+l\right) / 2 .
$$

If $p \equiv-1(\bmod 6)$, then $m \equiv n \equiv \frac{-p-1}{6}(\bmod p)$. Let

$$
m=-k p-\frac{p+1}{6} \text { and } n=-l p-(p+1) / 6
$$

we also have

$$
m(3 m+1) / 2+5 n(3 n+1) / 2=\left(p^{2}-1\right) / 4+p^{2}\left(3 k^{2}+k\right) / 2+5 p^{2}\left(3 l^{2}+l\right) / 2 .
$$

Extracting the terms whose power of $q$ is congruent to $\frac{p^{2}-1}{4}$ modulo $p$ from (22), and employing the above analysis, we obtain

$$
\sum_{n=0}^{\infty} b\left(p n+\frac{p^{2}-1}{4}\right) q^{p n+\frac{p^{2}-1}{4}}=\sum_{k, l=-\infty}^{\infty}(-1)^{k+l} q^{\left(p^{2}-1\right) / 4+p^{2}\left(3 k^{2}+k\right) / 2+5 p^{2}\left(3 l^{2}+l\right) / 2},
$$

which can be simplified to

$$
\sum_{n=0}^{\infty} b\left(p n+\frac{p^{2}-1}{4}\right) q^{n}=\sum_{k, l=-\infty}^{\infty}(-1)^{k+l} q^{p\left(3 k^{2}+k\right) / 2+5 p\left(3 l^{2}+l\right) / 2} .
$$

Applying Euler's pentagonal number theorem again, we derive that

$$
\sum_{n=0}^{\infty} b\left(p n+\frac{p^{2}-1}{4}\right) q^{n}=\left(q^{p} ; q^{p}\right)_{\infty}\left(q^{5 p} ; q^{5 p}\right)_{\infty},
$$

which completes the proof. 
Based on Lemma 10, we can easily obtain the following congruence.

Theorem 11. If $p \geqslant 5$ is a prime with $\left(\frac{-5}{p}\right)=-1$, we have

$$
\sum_{n=0}^{\infty} c_{5}\left(3 p n+\frac{3 p^{2}+1}{4}\right) q^{n} \equiv\left(q^{p} ; q^{p}\right)_{\infty}\left(q^{5 p} ; q^{5 p}\right)_{\infty} \quad(\bmod 3) .
$$

Proof. It follows from (8) and (20) that

$$
c_{5}(3 n+1) \equiv b(n) \quad(\bmod 3) .
$$

Applying Lemma 10, we deduce that

$$
\sum_{n=0}^{\infty} c_{5}\left(3\left(p n+\frac{p^{2}-1}{4}\right)+1\right) q^{n} \equiv\left(q^{p} ; q^{p}\right)_{\infty}\left(q^{5 p} ; q^{5 p}\right)_{\infty} \quad(\bmod 3)
$$

which finishes the proof.

One can generalize the above congruence to the form as we show below.

Theorem 12. Given a prime $p \geqslant 5$ with $\left(\frac{-5}{p}\right)=-1$, then for all $\alpha \geqslant 1$, we have

$$
\sum_{n=0}^{\infty} c_{5}\left(3 p^{2 \alpha-1} n+\frac{3 p^{2 \alpha}+1}{4}\right) q^{n} \equiv\left(q^{p} ; q^{p}\right)_{\infty}\left(q^{5 p} ; q^{5 p}\right)_{\infty} \quad(\bmod 3) .
$$

Proof. The proof follows by induction on $\alpha$. The case $\alpha=1$ is given in Theorem 11 . Assuming the result holds for a positive integer $\alpha=t$, namely,

$$
\sum_{n=0}^{\infty} c_{5}\left(3 p^{2 t-1} n+\frac{3 p^{2 t}+1}{4}\right) q^{n} \equiv\left(q^{p} ; q^{p}\right)_{\infty}\left(q^{5 p} ; q^{5 p}\right)_{\infty} \quad(\bmod 3) .
$$

Choosing those terms on each side whose power of $q$ is of the form $p n$, and replacing $q^{p}$ by $q$, we obtain

$$
\sum_{n=0}^{\infty} c_{5}\left(3 p^{2 t} n+\frac{3 p^{2 t}+1}{4}\right) q^{n} \equiv(q ; q)_{\infty}\left(q^{5} ; q^{5}\right)_{\infty} \quad(\bmod 3),
$$

which implies that

$$
c_{5}\left(3 p^{2 t} n+\frac{3 p^{2 t}+1}{4}\right) \equiv b(n) \quad(\bmod 3) .
$$

Furthermore, from Lemma 10 we see that

$$
\sum_{n=0}^{\infty} c_{5}\left(3 p^{2 t}\left(p n+\frac{p^{2}-1}{4}\right)+\frac{3 p^{2 t}+1}{4}\right) q^{n} \equiv\left(q^{p} ; q^{p}\right)_{\infty}\left(q^{5 p} ; q^{5 p}\right)_{\infty} \quad(\bmod 3),
$$

which upon simplification completes the induction on $\alpha$. 
As an immediate consequence of Theorem 12, we obtain the following infinite families of congruences for $c_{5}(n)$.

Corollary 13. Given a prime $p \geqslant 5$ with $\left(\frac{-5}{p}\right)=-1$, if $\alpha \geqslant 1$ and $n \geqslant 0$, we have

$$
c_{5}\left(3 p^{2 \alpha} n+3 p^{2 \alpha-1} i+\frac{3 p^{2 \alpha}+1}{4}\right) \equiv 0 \quad(\bmod 3)
$$

where $i=1,2, \ldots, p-1$.

Proof. Collecting those terms on each side of (24) for which the powers of $q$ are of the form $p n+i$, dividing by $q^{i}$, and replacing $q^{p}$ by $q$, we obtain that for $i=1,2, \ldots, p-1$,

$$
\sum_{n=0}^{\infty} c_{5}\left(3 p^{2 \alpha-1}(p n+i)+\frac{3 p^{2 \alpha}+1}{4}\right) q^{n} \equiv 0 \quad(\bmod 3),
$$

which proves the claim in the corollary.

\section{Acknowledgements}

The authors would like to thank an anonymous referee for a very careful review and many valuable comments on the manuscript. The first author was supported by the Young Doctor Development Foundation of 121 Talent Project of Central University of Finance and Economics (No. QBJ1402) and the second author was supported by the National Natural Science Foundation of China (No. 11401080).

\section{References}

[1] G.E. Andrews and B.C. Berndt. Ramanujan's Lost Notebook, Part I. Springer, 2000.

[2] B.C. Berndt. Ramanujan's Notebooks, Part III. Springer, 1991.

[3] K. Bringmann and J. Lovejoy. Rank and congruences for overpartition pairs. Int. J. Number Theory, 4:303-322, 2008.

[4] H.-C. Chan. Ramanujan's cubic continued fraction and an analog of his "most beautiful identity". Int. J. Number Theory, 6:673-680, 2010.

[5] H.-C. Chan. Ramanujan's cubic continued fraction and Ramanujan type congruences for a ceratin partition function. Int. J. Number Theory, 6:819-834, 2010.

[6] H.-C. Chan. Distribution of a certain partition function modulo powers of primes. Acta Math. Sin., 27:625-634, 2011.

[7] H.H. Chan and P.C. Toh. New analogues of Ramanujan's partition idenities. J. Number Theory, 130:1898-1913, 2010.

[8] S.H. Chan and R. Mao. Pairs of partitions without repeated odd parts. J. Math. Anal. Appl., 394:408-415, 2012. 
[9] S.C. Chen. Arithmetic properties of a partition pair function. Int. J. Number Theory, 10:1583-1594, 2014.

[10] W.Y.C. Chen and B.L.S. Lin. Congruences for bipartitions with odd parts distinct. Ramanujan J., 25:277-293, 2011.

[11] W.Y.C. Chen and B.L.S. Lin. Arithmetic properties of overpartition pairs. Acta Arith., 151:263-277, 2012.

[12] B. Kim. The overcubic partition function mod 3. Ramanujan Math. Soc. Lect. Notes Ser., 14:157-163, 2010.

[13] B. Kim. A crank analog on a certain kind of partition function arising from the cubic continued fraction. Acta Arith., 148:1-19, 2011.

[14] B.L.S. Lin. Arithmetic properties of bipartitions with even parts distinct. Ramanujan J., 33:269-279, 2014.

[15] B.L.S. Lin. Some results on bipartitions with 3-core. J. Number Theory, 139:44-52, 2014.

[16] B.L.S. Lin. An infinte familiy of congruences modulo 3 for 13-regular bipartitions. Ramanujan J., 2014. doi:10.1007/s11139-014-9610-7

[17] B.L.S. Lin. Arithmetic of the 7-regular bipartition function modulo 3. Ramanujan J., 2014. doi:10.1007/s11139-013-9542-7

[18] J. Sinick. Ramanujan congruences for a class of eta quotients. Int. J. Number Theory, 6:835-847, 2010.

[19] P.C. Toh. Ramanujan type identities and congruences for partition pairs. Discrete Math., 312:1244-1250, 2012.

[20] X.H. Xiong. The number of cubic partitions modulo powers of 5. Sci Sin Math., 41:1-15, 2011. 